\title{
Energy-Efficient Node Scheduling Method for Cooperative Target Tracking in Wireless Sensor Networks
}

\author{
Weirong Liu, Yun He, Xiaoyong Zhang, Fu Jiang, Kai Gao, and Jianming Xiao \\ School of Information Science and Engineering, Central South University, Changsha 410075, China \\ Correspondence should be addressed to Xiaoyong Zhang; zhangxy@csu.edu.cn
}

Received 25 July 2014; Revised 13 October 2014; Accepted 15 October 2014

Academic Editor: Wei Zhang

Copyright ( 2015 Weirong Liu et al. This is an open access article distributed under the Creative Commons Attribution License, which permits unrestricted use, distribution, and reproduction in any medium, provided the original work is properly cited.

\begin{abstract}
Using the sensor nodes to achieve target tracking is a challenging problem in resource-limited wireless sensor networks. The tracking nodes are usually required to consume much energy to improve the tracking performance. In this paper, an energy-efficient node scheduling method is proposed to minimize energy consumption while ensuring the tracking accuracy. Firstly, the Kalmanconsensus filter is constructed to improve the tracking accuracy and predict the target position. Based on the predicted position, an adaptive node scheduling mechanism is utilized to adjust the sample interval and the number of active nodes dynamically. Rather than using traditional search algorithm, the scheduling problem is decomposed to decouple the sample interval and number of nodes. And the node index is mapped into real domain to get closed-form solution to decide the active nodes. Thus, the NP-complete nature is avoided in the proposed method. The proposed scheduling method can keep the tracking accuracy while minimizing energy consumption. Simulation results validate its effective performance for target tracking in wireless sensor networks.
\end{abstract}

\section{Introduction}

Wireless sensor network (WSN), which consists of tiny lowcost, energy-limited, and sensing range-limited nodes, has received extensive research in recent years. The nodes in WSN, equipped with one or more sensors, can sense, measure, and gather information from vicinal area. By utilizing the wireless RF module, these nodes can transmit the gathered information from local region to remote base station through node's multiple-hop relay. With the development of microelectronic technology, WSN has been deployed in various application scenarios to observe physical environmental change and detect events of interest [1].

In all kinds of practical scenarios, target tracking is one of the most important applications of WSN. Target tracking is a process of estimating or predicting the trajectories and velocities of some mobile targets by the sensor nodes in WSN collaboratively. The cooperation among sensor nodes could improve the accuracy of target's location or velocity. The targets of tracking can be any mobile objects, such as animals, humans, and vehicles [2].

With the development of WSN, numerous target tracking applications have emerged in many practical projects. For instance, PinPtr [3] is a counter-sniper system applied in military field to detect and locate enemy shooters. Underwater monitoring system in [4] developed a submarine platform to monitor coral reefs and fisheries. CenWits [5] is a search-andrescue system utilizing static or mobile sensor nodes to locate stranded person in wreckage. ZebraNet system [6] consists of sensor nodes installed in animals to track and research the migration of wildlife populations. These projects have been used intensively in environmental detecting, industrial monitoring, disaster alerting, and healthcare [2].

Differing from the traditional target tracking, the target tracking using WSN brings up many challenges: keeping the tracking accuracy under the node's resources constraints. As most existing works have mentioned, the constraints of node resources, such as sensing range, communication bandwidth, and computation ability, are critical factors to keep accuracy and to save energy for target tracking in wireless sensor networks [7]. Recently, many researches have proposed algorithms to improve energy efficiency while keeping the target tracking accuracy.

Some researches focus on reducing the communication cost in target tracking. In [8], a heuristic algorithm to construct an efficient object tracking in wireless sensor networks 
was developed, which formulates the minimizing communication cost as $0 / 1$ integer programming problem, and a Lagrangian relaxation based heuristic algorithm was proposed to solve this optimization problem. In [9], a publishand-subscribe method and drain-and-balance policy were described, respectively, to optimize the structure of network communication and reduce communication consumption. Reference [10] was considered to adjust physical topology of the sensor network, so the total communication cost was reduced. Although these works were devoted to reducing the energy consumption, they have limitation on network energy saving by just minimizing the communication cost, and they may also not be scalable enough.

Recently much attention has been focusing on sensor node scheduling to reduce energy for target tracking. The node scheduling can be classified into 2 categories: the random selection method and adaptive selection method. In random selection method, the sensing nodes are randomly selected according to a certain degree of probability; in adaptive selection method, the sensing nodes are selected according to the critical factors such as node type, detecting ability, and residual energy.

Random selection method has compared low scheduling cost and it is easy to deploy in real WSN. In [11], a probabilistic scheduling of the duty cycle of the sensors was provided in a sensor network deployed in an area of interest based on a Poisson distribution. Its tracking algorithm exploits signal from multiple sensor nodes in several modalities, relying on prior statistical information about target models. In [12], the authors describe the key ideas behind the CSP algorithms for distributed sensor networks and present how the CSP algorithms interface with the networking/routing algorithms. An entropy-based sensor selection heuristic algorithm for location-to-location was proposed in [13], which needs (1) a prior probability distribution of the target location and (2) the locations and the sensing models of a set of candidate sensors for selection. These works concentrated on improving the energy conservation by randomly selecting tasking sensors.

However, sensors' random sleep with a probability may not keep the target tracking accuracy because some sensors close to a target may be in sleep mode. Even in the target sensing region, there are not active nodes. But it is also sufficiently important to keep the performance of the target tracking. From this point of view, node selection along with the trajectory of moving target has aroused much interest. Some practical distributed sensor node selection algorithms have been proposed to improve energy efficiency with reliable tracking [14-18].

In [14], an energy-efficient selection of cooperative nodes was presented. According to the information utility and the remaining energy of sensor nodes, the authors in [10] constructed an objective function and proposed a dynamic node selection scheme based on genetic algorithms. Although the simulation results have shown its good effect, the node selection scheme based on genetic algorithms is difficult to apply in real applications and may not be suitable for the realtime requirement. The authors in [15] proposed an energyefficient distributed adaptive multisensor scheduling for target tracking. The number of tasking sensors and the sampling time interval are taken into consideration. To select the tasking node, the leader needs to know its target detection probability which can be deduced from the target state equations. But this process may be somehow complex and requires implementing the Monte Carlo method. An adaptive sensor scheduling is formulated in [16], which contains two tracking modes: the fast tracking mode and the tracking maintenance mode. The energy conservation was achieved by adaptively adjusting the sampling time interval. But it is only applied to selected single tasking sensor at each sample interval. In $[17,18]$, the variable sampling interval was also adopted, but it cannot realize the joint optimization to energy.

Summarizing the above works, the main factors that influence position accuracy and energy efficiency of target tracking include the network communication topology, the sampling time interval, and the number of tasking sensors. The number of tasking sensors is directly related to the total energy consumption in tracking process. However, the current adaptive node selection method could not permit large candidate node set because of their high complexity.

Comprehending these factors, this paper aims to propose a novel node scheduling method with cooperative Kalmanconsensus filter to reduce the energy consumption while keeping tracking accuracy. The Kalman-consensus filter is used to obtain the target state estimation and predict the next step position. The node selection problem is transformed into a convex optimization problem, which is decomposed, and a Lagrangian function is used to solve it.

The main contributions of this paper include (1) extending the classic Kalman filter to cooperative form, which can combine the local nodes' information to improve the tracking precision; (2) proposing a joint sample interval and node selection optimization scheme, which can realize the energy consumption minimum while keeping the tracking accuracy; (3) addressing the NP-hard joint optimization problem, adopting a map method to map the selecting factor to real domain; and utilizing gradient information to get the solution rapidly.

The rest of this paper is organized as follows. The problem formulation, dynamic model, and energy model are analyzed in Section 2. In Section 3, the novel node selection method is presented. Simulation results are proposed in Section 4. Finally, conclusion and future work are given in Section 5.

\section{Problem Formulation}

2.1. Target Model and Kalman-Consensus Filter (KCF). Considering that wireless sensor network is constructed by deploying $n$ sensor nodes and a moving target, all cognitive sensors have the same sensing range and can jointly capture the target trajectory. The target model is taken as general linear model, like in [17]. A moving target with the system disturbance is described by the differential equation

$$
x(k+1)=A(x(k)) x(k)+B \omega(k),
$$

where $x(k)=\left(q_{x}(k), p_{x}(k), q_{y}(k), p_{y}(k)\right)^{T}$ denotes the state of the target at time $k ; q=\left(q_{x}, q_{y}\right)^{T}$ and $p=\left(p_{x}, p_{y}\right)^{T}$ are 
the coordinate and velocity of the target, respectively, in twodimensional coordinate system; and $\omega(k)$ is the zero-mean Gaussian white noise with variance $\sigma_{0}^{2}$, which is the process noise.

The state matrix $A(x)$ is depicted as

$$
\begin{gathered}
A(x, \Delta t)=F(x) \otimes M_{1}(\Delta t)+\left(I_{2}-F(x)\right) \otimes M_{2}, \\
M_{1}=\left[\begin{array}{ll}
1 & \varepsilon \\
0 & 1
\end{array}\right], \quad M_{2}=\left[\begin{array}{cc}
1 & \varepsilon \\
-\varepsilon c_{1} & 1-\varepsilon c_{2}
\end{array}\right], \\
F(x)=\left[\begin{array}{cc}
\mu\left(x_{1}\right) & 0 \\
0 & \mu\left(x_{3}\right)
\end{array}\right],
\end{gathered}
$$

where $\varepsilon$ is the step size and does not depend on the sampling time interval, which means that the target's motion is independent of the sensor's sampling frequency. $\mu(x)$ is defined by

$$
\begin{gathered}
\mu(x)=\frac{\rho(a+x)+\rho(a-x)}{2}, \\
\rho(x)= \begin{cases}1, & x \geq 0, \\
-1, & x<0 .\end{cases}
\end{gathered}
$$

The matrix $B$ is defined as

$$
B=I_{2} \otimes G, \quad G=\left[\begin{array}{c}
\frac{\varepsilon^{2} \sigma_{0}}{2} \\
\varepsilon \sigma_{0}
\end{array}\right],
$$

where $c_{1}, c_{2}>0$ are the parameters of a PD controller and " $\otimes$ " denotes the Kronecker product of matrix.

The measurement model is given by

$$
z_{i}(k)=H x(k)+v_{i}(k)
$$

where $x(k)$ is the state of the target, $H$ is the observation model, and $v_{i}$ is the measurement zero-mean Gaussian white noise of the sensor $i$ with covariance $R_{i}$.

The Kalman-consensus filter (KCF) algorithm used in this paper is mainly referred to in [19] and operates as shown in Algorithm 1.

2.2. Sensor Detection Model. The binary detection model, described in most of the existing works $[14,20]$, assumes that if the target is within the sensing zone of a node, the detection of the target is successful. This is an ideal assumption to sensor's detection ability. Generally, there is always uncertainty when sensors perform the operation of detection. In this paper, the target probability detected by node $s_{i}$ is

$$
p\left(s_{i}\right)= \begin{cases}0, & r_{s}+r_{e} \leq d\left(s_{i}, P\right), \\ e^{-\lambda \alpha^{\beta}}, & r_{s}-r_{e} \leq d\left(s_{i}, P\right)<r_{s}+r_{e}, \\ 1, & r_{s}-r_{e} \geq d\left(s_{i}, P\right),\end{cases}
$$

where $r_{e}\left(r_{e}<r\right)$ is the uncertainty measurement length of node $s_{i}, \alpha=d\left(s_{i}, P\right)-\left(r-r_{e}\right)$, and $\alpha$ and $\beta$ are measurement parameters dependent on sensor type.

Based on this sensing model, for a target located in $P(x, y)$ detected by $m$ sensors $s_{1}, s_{2}, \ldots, s_{m}$, the joint detection probability for these $m$ tasking sensors is given by

$$
P_{d}=1-\prod_{i=1}^{m}\left(1-p\left(s_{i}\right)\right)
$$

2.3. Tracking Error. According to [21], the estimated target state error covariance at $k$ time step can be defined as

$$
P(k \mid k) \equiv\left[\sigma_{i j}\right]
$$

and the predicted target state error covariance can be calculated as

$$
P(k \mid k)=\sum\left(\Delta t_{k}\right)=\left[\begin{array}{cc}
\sigma_{11}+2 \Delta t_{k} \sigma_{12}+\Delta t_{k}^{2} \sigma_{22} & \sigma_{13}+\Delta t_{k}\left(\sigma_{14}+\sigma_{23}\right)+\Delta t_{k}^{2} \sigma_{24} \\
\sigma_{13}+\Delta t_{k}\left(\sigma_{14}+\sigma_{23}\right)+\Delta t_{k}^{2} \sigma_{24} & \sigma_{33}+2 \Delta t_{k} \sigma_{34}+\Delta t_{k}^{2} \sigma_{44}
\end{array}\right] .
$$

The predicted target state uncertainty is described as follows:

$$
\begin{aligned}
\phi(k+1 \mid k)= & \operatorname{trace}\left(\sum\left(\Delta t_{k}\right)\right) \\
= & \left(\sigma_{11}+\sigma_{33}\right)+\left(2 \sigma_{12}+2 \sigma_{34}\right) \Delta t_{k} \\
& +\left(\sigma_{22}+\sigma_{44}\right) \Delta t_{k}^{2}+\frac{2}{3} q \Delta t_{k}^{3} .
\end{aligned}
$$

A threshold $\phi_{0}$ is preset as the tracking accuracy. If the predicted target state uncertainty $\phi(k+1 \mid k)$ is less than $\phi_{0}$, the tracking accuracy is supposed to be satisfied. Otherwise, it is deemed to be unsatisfied and needs to be improved.
2.4. Energy Model. At each tracking step, as the energy model in [22] shows, energy cost is mainly used in target detecting, data sending, data receiving, and data processing.

If the current tasking node $i$ selects node $j$ as the candidate sensor for the next tracking step, the energy cost by sending data from node $i$ to node $j$ is $E_{t}(i, j)=\left(e_{t}+e_{d} r_{i j}^{\alpha}\right) b_{c}$, where $e_{t}$ and $e_{d}$ are decided by the transmitter and $r_{i j}$ is the distance between sensors $i$ and $j . \alpha$ is known parameter that relies on channel characteristic, and $b_{c}$ is the number of bits of the transmitted data.

The energy cost in receiving data by sensor node $j$ is $E_{r}(j)=e_{r} b_{c}$, where $e_{r}$ is decided by receiver installed in sensor node $j$. In a practical application, energy consumption mainly comes from communication between sensors. Hence, 
Given the initial parameters $P_{i}=P_{0}, \bar{x}_{i}=x(0)$, for sensor node $i$ at time step $k$

(1) Obtain measurement $z_{i}$ with covariance $R_{i}$.

(2) Compute information vector and matrix of node $i$

$$
\begin{aligned}
& u_{i}=H_{i}^{T} R_{i}^{-1} z_{i} \\
& U_{i}=H_{i}^{T} R_{i}^{-1} H_{i} .
\end{aligned}
$$

(3) Send message $m_{i}=\left(u_{i}, U_{i}, \bar{x}_{i}\right)$ to active neighbors.

(4) Receive messages from all active neighbors.

(5) Fuse information matrices and vectors

$$
y_{i}=\sum_{j \in J_{i}} u_{j}, \quad S_{i}=\sum_{j \in J_{i}} U_{j} .
$$

(6) Compute the Kalman-consensus state estimation

$$
\begin{aligned}
& M_{i}=\left(P_{i}^{-1}+S_{i}\right)^{-1}, \\
& \hat{x}_{i}=\bar{x}_{i}+M_{i}\left(y_{i}-S_{i} \bar{x}_{i}\right)+\xi M_{i} \sum_{j \in N_{i}}\left(\bar{x}_{j}-\bar{x}_{i}\right), \\
& \xi=1 /\left(\left\|M_{i}\right\|+1\right) .
\end{aligned}
$$

(7) Update the estimate state of the target

$$
\begin{aligned}
P_{i} & \leftarrow A M_{i} A^{T}+B Q B^{T} \\
\bar{x}_{i} & \leftarrow A \widehat{x}_{i}
\end{aligned}
$$

Algorithm 1: Kalman-consensus filter.

we treat the energy cost from sensing and processing as a union; that is, $E_{s}(j)=e_{s} b_{s}$. Let $E_{i}(k)$ be the remaining energy after $k-1$ time step that is also the available energy at $k$ tracking step. Hence, the total energy cost for tasking sensor $i$ is

$$
E(j)=E_{t}(i, j)+E_{r}(j)+E_{s}(j)=e_{0}+e_{1} r_{i j}^{\alpha},
$$

where

$$
\begin{gathered}
e_{0}=\left(e_{t}+e_{r}\right) b_{c}+e_{s} b_{s}, \\
e_{1}=e_{d} b_{c} .
\end{gathered}
$$
by

And the total energy consumed for a tracking step is given

$$
E_{T}=\sum_{i=1}^{N} \rho_{i}\left(e_{0}+e_{h i}^{\alpha}\right)
$$

where $\rho_{i} \in\{0,1\}$ is the assignment index and it denotes by " 1 " being active to track target and by " 0 " sleeping to save energy. As the cluster header, the energy consumption for estimating state fusion and selecting the next tasking sensors as well as transmitting $\widehat{x}_{t}$ to receivers is

$$
E(h)=\sum_{i \in G(k+1)-s_{h}}\left(e_{t}+e_{d} r_{h i}^{\alpha}+e_{r}\right) b_{c},
$$

where $G(k)$ represents the sensor cluster at $k$ tracking step and $s_{k}$ is the cluster header.

\section{Adaptive Node Scheduling Method}

3.1. Analysis of Target Tracking Process. Figure 1 shows a general target tracking scene in a wireless sensor network with uniform deployment sensor nodes. Sensor nodes will be active on demand following the target trajectory. To save energy, not all of the sensors in sensing zone are selected to detect the target. These selected sensors, at first, cooperate to generate an estimation of target position using Kalmanconsensus filter algorithm. And then they send their estimation to cluster header for further fusion so that more accurate estimation of target position is achieved. The goal of the method is twofold: (i) generating the state estimation and predicting the position of dynamic target and (ii) selecting the tasking cluster and cluster header for the tracking step.

At the beginning of detecting, all sensors are in the sleep state initially, except for sensors that are on the borders of the sensor filed. The sensor nodes on borders that first found the target will broadcast the target information and start the tracking task. They will obtain the first measurement and calculate the target state estimation to select and activate the next tasking sensor nodes (including the cluster header) for the next sample interval. They will send their state prediction to the next tasking cluster.

At $k$ tracking step, the selected sensor nodes in current active cluster perform the following sensing tasks:

(1) obtaining measurement $z(k)$ of target location with $R$;

(2) computing and updating the state estimation using Kalman-consensus filter;

(3) sending the updated state estimation $(P, \bar{x})$ to cluster header.

As for the cluster header, except for the above tasks, it needs to perform the following additional jobs:

(1) fusing the state estimation $(P, \bar{x})$ so that an accurate estimation of target position is achieved;

(2) calculating the sampling interval $\Delta t$ for $k+1$ tracking step;

(3) selecting the tasking cluster for the step $k+1$;

(4) selecting a new cluster header for the new tasking cluster;

(5) transmitting the fused target estimation $\widehat{x}_{t}$ to the next tasking sensor nodes.

Once the sensor nodes in the tasking cluster at step $k$ receive the estimation information from their cluster header to next cluster, they will turn into sleep mode at step $k+1$.

3.2. Adaptive Node Selection Combining with KalmanConsensus Filter Algorithm (ANS-KCF). For the problem of node selection for distributed cooperative target tracking, the key issue is to form tasking sensor cluster dynamically, which directly related to energy consumption and tracking accuracy. The objective of proposed method is to minimize the energy cost of network under the condition of desired tracking error. In addition, the sampling time interval has a great influence on tracking accuracy and network energy cost. Actually, if the desired tracking performance is obtained, a bigger sampling time interval will be a better choice for energy saving. 


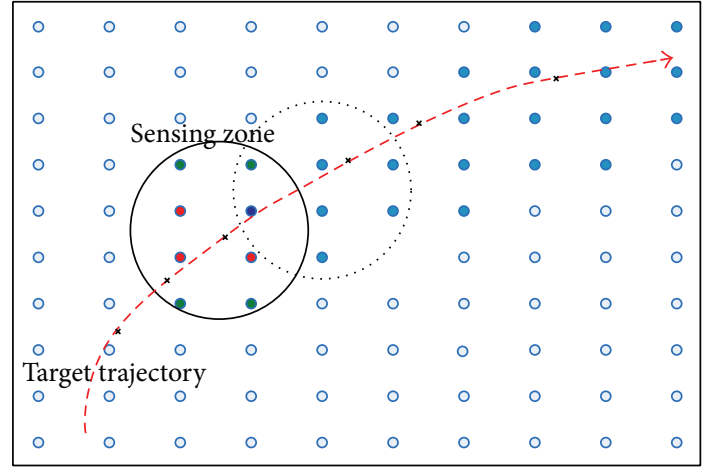

- Current candidate sensors

- Current selected sensors

- Future active sensors

- Cluster head

* Estimated target position

FIGURE 1: Target tracking scene in WSN.

The formation of next cluster candidate set includes two phases: the first phase is the target tracking, in which nodes that can detect the target are active; the second phase is the detection probability $p\left(s_{i}\right)$ calculating, which is calculated according to (6). Only these nodes whose detection probability is bigger than the threshold $\theta$ can be taken as the candidates. Hence, the set of candidate sensor nodes can be given by

$$
V=\left\{s_{i} \mid p\left(s_{i}\right)>\varepsilon, \operatorname{dis}\left(s_{i}, \widehat{x}_{t}\right)<r_{s}\right\},
$$

where $\widehat{x}_{t}$ is the estimate position at $k$ tracking step.

In order to better express the node selecting problem, the joint detection probability (7) to the following form is

$$
P_{d}=1-\prod_{i=1}^{V}\left(1-\rho_{i} p\left(s_{i}\right)\right) \text {. }
$$

Based on the sensor detection probability model and the energy model given previously, the node selection problem can be formulated as follows: at tracking step $k$, considering the target state estimation $\hat{x}_{t}$, its error covariance $P(k \mid k)$, and the sampling time interval $\Delta t_{k}$, the tasking sensors at tracking step $k+1$ are determined such that

$$
\begin{aligned}
& \min _{\rho_{i}, \Delta t_{k}} \quad J=\frac{1}{\Delta t_{k}} \sum_{i=1}^{|V|} \rho_{i}\left(e_{0}+e_{1} r_{h i}^{\alpha}\right) \\
& \text { subject to: } \phi(k+1 \mid k) \leq \phi_{0}, \quad P_{d} \geq \theta_{d}
\end{aligned}
$$

where $\theta_{d}$ is the preset joint detection threshold for multiple sensor nodes.

Because of the complexity in computing the detection probability $P_{d}$ and the coupling effect of the sampling interval $\Delta t_{k}$ and the selection of the tasking sensor nodes, a two-stage suboptimal algorithm is designed to approximate the original optimization problem (17).

As mentioned above, the fixed sampling time interval is not suitable for energy-efficient target tracking. We suppose that $\Delta t_{k}$ is in the range $\left[T_{\min }, T_{\max }\right]$, where $T_{\min }$ is the given minimal sampling intervals and cannot be too small because it must be larger than the total duration span for tracking activities. A maximum sampling interval $\Delta t_{k}$ is obtained to satisfy the given tracking accuracy specification $\phi_{0}$. The corresponding suboptimization problem is

$$
\begin{array}{ll}
\max & \left(\Delta t_{k}\right) \\
\text { s.t. } & \phi(k+1 \mid k) \leq \phi_{0} .
\end{array}
$$

According to [15], the max $\Delta t_{k}$ will be obtained at the constraint bound:

$$
\phi(k+1 \mid k)=\phi_{0} .
$$

By solving the following equation, we can get the suitable $\Delta t_{k}^{*}$. That is,

$$
\begin{aligned}
& \Delta t_{k}^{*}=\underset{\Delta t_{k}}{\arg \max }\left(\Delta t_{k}\right) \\
& \text { s.t. } \phi(k+1 \mid k) \leq \phi_{0} .
\end{aligned}
$$

At the second stage, a number of sensors are selected based on the determined sampling interval $\Delta t_{k}^{*}$ to form a temporary tasking cluster to achieve the target detection probability threshold. Therefore (17) can be rewritten as the decomposed problem; the second suboptimization problem can be expressed in the following mathematical form:

$$
\begin{array}{ll}
\min _{\rho_{i}} & J=\frac{1}{\Delta t_{k}^{*}} \sum_{i=1}^{V} \rho_{i}\left(e_{0}+e_{1} r_{h i}^{\alpha}\right) \\
\text { s.t. } & P_{d} \geq \theta_{d} .
\end{array}
$$

Since $\partial J / \partial \rho_{i}>0$ and $\partial P_{d} / \partial \rho_{i}>0$, also $\partial^{2} J / \partial \rho_{i}^{2}=0$ and $\partial^{2} P_{d} / \partial \rho_{i}^{2}=0$. This can be solved systematically as a convex optimization problem [23] where index and constraints are convex with respect to $\rho_{i}$.

Then the resulting convex problem can be solved to find the minimum $J$ and its corresponding parameters $\rho_{i}$ for each node and for networks. We use the Lagrangian function as follows:

$$
L\left(\rho_{i}, \lambda\right)=\sum_{i=1}^{V} \rho_{i}\left(e_{0}+e_{1} r_{h i}^{\alpha}\right)-\lambda\left(P_{d}-\theta_{d}\right)
$$

where $\lambda$ is the Lagrangian multiplier for the constraint in (21). Then the gradient for the Lagrangian function is

$$
\frac{\partial L}{\partial \rho_{i}}=\left(e_{0}+e_{1} r_{h i}^{\alpha}\right)-\lambda p\left(s_{i}\right) \prod_{k !=i}\left(1-\rho_{k} p\left(s_{k}\right)\right), \quad i \in V
$$

where $V$ is the candidate sensor set described by (15). To select the suitable sensing nodes, there is no need to solve this equation, which is complex. The goal of proposed method is just to determine the priority of the candidate sensor nodes for target tracking. From the Karush-Kuhn-Tucker conditions and the gradient (23), we let

$$
\frac{\partial L}{\partial \rho_{j}}=\left(e_{0}+e_{1} r_{h j}^{\alpha}\right)-\lambda p\left(s_{j}\right) \prod_{k !=j}\left(1-\rho_{k} p\left(s_{k}\right)\right)=0 .
$$


Then, for node $i$, the following can be obtained:

$$
\frac{\left(e_{0}+e_{1} r_{h i}^{\alpha}\right)}{\prod_{k !=i, j}\left(1-\rho_{k} p\left(s_{k}\right)\right)}-\lambda p\left(s_{i}\right)=-\lambda p\left(s_{i}\right) \rho_{j} p\left(s_{j}\right) .
$$

And for node $j$, the following can be obtained:

$$
\frac{\left(e_{0}+e_{1} r_{h j}^{\alpha}\right)}{\prod_{k !=i, j}\left(1-\rho_{k} p\left(s_{k}\right)\right)}-\lambda p\left(s_{j}\right)=-\lambda p\left(s_{j}\right) \rho_{i} p\left(s_{i}\right) \text {. }
$$

Therefore, the priority ratio (which is converse to the cost ratio) for node $i$ and node $j$ can be obtained as follows:

$$
\begin{aligned}
\frac{\rho_{i}}{\rho_{j}} & =\frac{\left(e_{0}+e_{1} r_{h j}^{\alpha}\right)-\lambda p\left(s_{j}\right) \prod_{k \neq i, j}\left(1-\rho_{k} p\left(s_{k}\right)\right)}{\left(e_{0}+e_{1} r_{h i}^{\alpha}\right)-\lambda p\left(s_{i}\right) \prod_{k \neq i, j}\left(1-\rho_{k} p\left(s_{k}\right)\right)} \\
& \triangleq \frac{\cos t(j)}{\cos t(i)} .
\end{aligned}
$$

From (27), the cost function can be defined as

$$
\cos t(i)=\left(e_{0}+e_{1} r_{h i}^{\alpha}\right)-\lambda p\left(s_{i}\right) \prod_{k !=i, j}\left(1-\rho_{k} p\left(s_{k}\right)\right) .
$$

The cost function is the energy consumption under the target detection constraint if the nodes are selected.

The next stage is to determine the optimum $\lambda$ and to select the sensor nodes for target tracking. The node with max remaining energy will be selected firstly and it can be taken as cluster header. Then, considering just two nodes (i.e., the other sensors are not selected yet), (26) can be simplified as

$$
\cos t(i)=e_{0}+e_{1} r_{h i}^{\alpha}-\lambda p\left(s_{i}\right) .
$$

The optimal $\lambda$ should be calculated and it may be a positive nonzero parameter or a zero parameter. Consider the complimentary slackness conditions:

$$
\begin{aligned}
& \lambda\left(P_{d}-\theta_{d}\right)=0 \longrightarrow \lambda=0, \quad P_{d}>\theta_{d}, \\
& \lambda\left(P_{d}-\theta_{d}\right)=0 \longrightarrow \lambda \neq 0, \quad P_{d}=\theta_{d} .
\end{aligned}
$$

If $\lambda=0$ is the optimal solution, then, due to (30a), $P_{d}>\beta$ is satisfied. We also know that $P_{d}$ is the increasing functions of $\rho_{j}$. Therefore, we can decrease $\rho_{j}$ so that $P_{d}=\beta$ is satisfied. Under this reduction, we have smaller $P f$ and $e_{0}+e_{1} r_{h i}^{\alpha}$, which leads to a more desirable answer. In this way, $\lambda$ is selected so that $P_{d}=\theta_{d}$ is satisfied. It is equal to (30b) condition. Thus, in this paper, the optimal $\lambda$ is positive nonzero parameter and there is $P_{d}=\theta_{d}$.

In order to find the optimum $\lambda$ we use an iterative bisection algorithm. We search through the algorithm to satisfy the optimal conditions stated before. At each iteration, cost functions $\cos t(*)$ for all sensors in (29) are calculated and sorted in ascending order. Then the sensors with the highest priority are selected until the global $P_{d}>\beta$ is satisfied. Then $\lambda$ is updated according to the computed $P_{d}$ and searching space is halved and the algorithm is repeated again.

This iterative algorithm ends when the accuracy of $\lambda$ becomes smaller than $\varepsilon$. $\varepsilon$ declares the resolution of the proposed algorithm. Noting that in each iteration, to obtain the optimal $\lambda$, if $P_{d}>\theta_{d}$, then $\lambda$ is decreased and $P_{d}<\theta_{d}$; then $\lambda$ is increased. It means that the proposed algorithm converges to the optimal $\lambda$ which satisfied $P_{d}=\theta_{d}$.

Because the solving of optimum $\lambda$ adopts bisection iteration, its order is $\left(\log _{2}\left(\lambda_{\max }-\lambda_{\min }\right) / \varepsilon\right)$. For each iteration, the proposed algorithm has the linear complexity with the order of $O(N)$, since the cost functions for all sensors are computed. Thus, to solve the optimal nodes' schedule design, the complexity is $\left(N \cdot \log _{2}\left(\lambda_{\max }-\lambda_{\min }\right) / \varepsilon\right)$. Then the optimal sample period is decided by iteration, which is a linear process with the order $N_{T}$. Because of the decoupling of node schedule and sample period in the proposed algorithm, the entire complexity of algorithm is $\left(N \cdot \log _{2}\left(\lambda_{\max }-\right.\right.$ $\left.\lambda_{\min }\right) / \varepsilon+N_{T}$ ). It must be mentioned that the cost of algorithm is mainly computing cost, whose energy consumption is minor compared to the energy consumption when the node participates in the target tracking.

In order to limit the search space of optimal $\lambda$, it is proper to find the $\lambda_{\max }$, which should guarantee the condition $0<$ $\lambda_{\text {optimal }}<\lambda_{\max } ; \lambda_{\max }$ is obtained when priorities of selecting sensors are determined according to their $p\left(s_{i}\right) s$. We sort $p\left(s_{i}\right) s$ for all nodes; then the relation between cost functions of two nodes becomes

$$
e_{\mathrm{amp}} s_{i}^{2}-\lambda_{\max } p\left(s_{i}\right)<e_{\mathrm{amp}} s_{i-1}^{2}-\lambda_{\max } p\left(s_{i-1}\right) \quad \forall i \in V .
$$

Hence,

$$
\lambda_{\max }>\frac{e_{\mathrm{amp}}\left(s_{i}^{2}-s_{i-1}^{2}\right)}{p\left(s_{i \max }\right)-p\left(s_{i-1 \max }\right)} \quad \forall i \in V .
$$

It means that $\lambda_{\max }$ should be selected according to (32) such that a suitable searching space is considered to find desirable answer.

Each node with the smaller cost function defined as (29) has the higher priority in target tracking. To determine the priority of tasking nodes, cost function in (29) is calculated for all of candidates in $V$ and sorted in ascending order. Algorithm 2 is given to calculate the optimal $\lambda$ and to select the tasking nodes from candidate sensor set.

\section{Simulation Results}

The intruder detection and tracking system in military is a representative application of target tracking. To avoid the sudden attack or surreptitious scout of enemy, the wireless sensor network is deployed in the buffer region between defensive line and the enemy. When the enemy combatants or vehicles enter the buffer region, the sensor network can detect these events and report the enemy position real time so that the troops can respond immediately.

To evaluate the performance of the proposed algorithm, the software MATLAB is used to simulate the intruder detection and tracking scene. The network scene is formed by sensing range-limited sensors and the monitoring area is $100 \mathrm{~m} \times 100 \mathrm{~m}$ with coordinates from $(-50,-50)$ to $(50,50)$, as shown in Figure 1. In the scene, a single moving target whose dynamics is given in (1) is tracked by 100 uniform distributed 


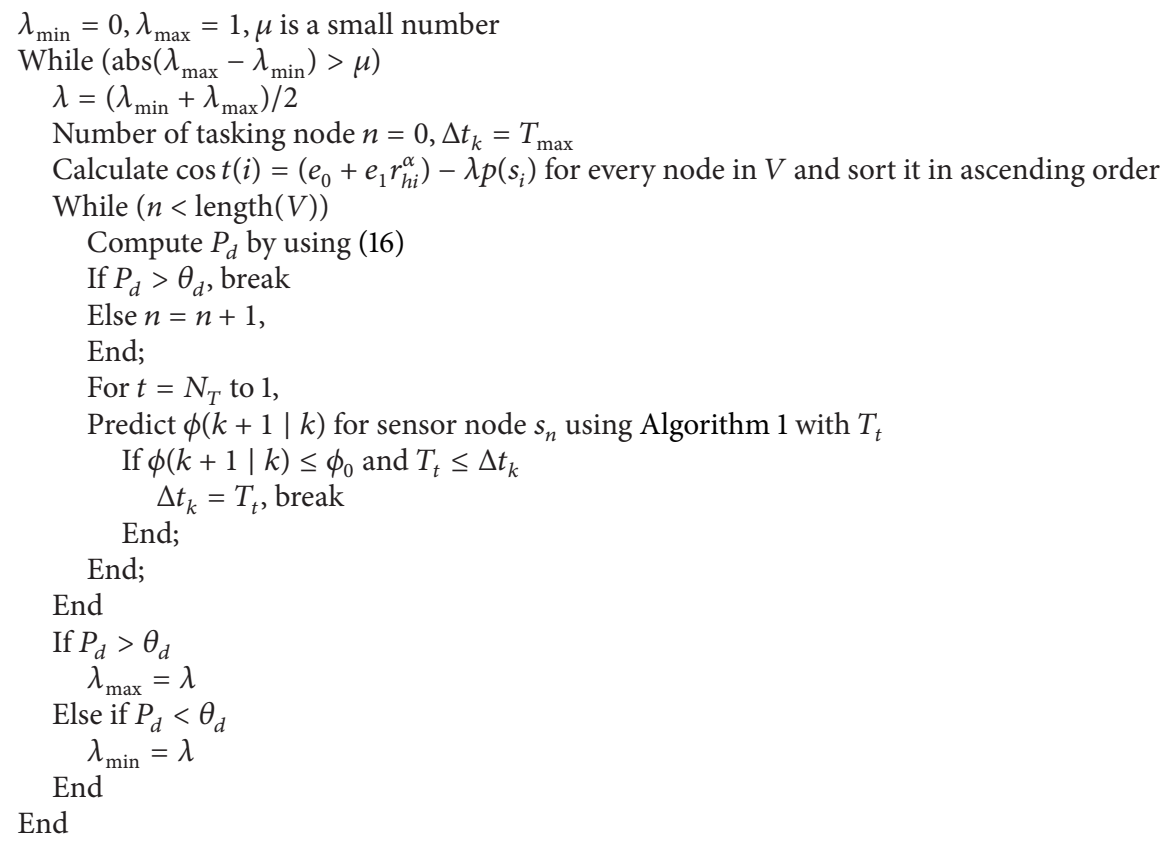

Algorithm 2: Adaptive node selection algorithm combining with Kalman-consensus filter (ANS-KCF).

sensor nodes. Each sensor node measures the position of the target in a $2 \mathrm{D}$ plane; that is,

$$
H=\left[\begin{array}{llll}
1 & 0 & 0 & 0 \\
0 & 0 & 1 & 0
\end{array}\right] .
$$

It is assumed that all the sensors in the network have the same sensing parameters; that is, the sensing and communication range of each sensor are $r_{s}=15$ and $r_{c}=18$, respectively. The measurement and process noise statistics are $R_{i}=9 I_{2}$ and $Q=25 I_{4}$, respectively. The desired sensor detection threshold is assumed to be $\theta_{d}=0.95$. The threshold of the tracking accuracy is also assumed to be $\phi_{0}=5$.

Firstly, the proposed method is tested and verified by comparing the estimated trajectory with real target trajectory. Figure 2 shows the estimated target trajectory of the proposed method. The target moves in sensing area for $40 \mathrm{~s}$ and forms a trajectory shown in Figure 2. It displays that the estimated trajectory is closely following the target trajectory. Furthermore, the tracking accuracy is shown in Figure 3. Obviously, the excellent tracking performance is obtained in simulation when using the proposed methods.

To display the performance more convincingly, the proposed tracking algorithm Kalman-consensus filter (KCF) is compared with EKF, and the accuracy of target tracking is evaluated using estimated trajectory and estimated error. Figures 4 and 6 list the estimated value of $X$ coordinate and $Y$ coordinate, respectively. And the corresponding estimated errors are shown in Figures 5 and 7, respectively.

From the deviation of estimated position and actual position described in Figures 4-7, both the proposed KCF and EKF can track target with small estimated error when target movement direction remains the same. But when the target

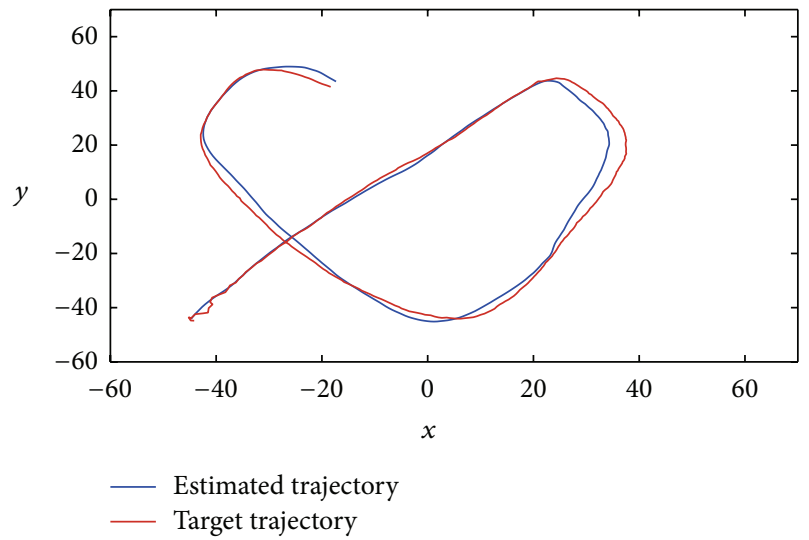

FIGURE 2: The comparison of estimated trajectory with the real target tracking trajectory.

direction changes, especially in the beginning of changes, the estimated trajectory with KCF is more close to the real target trajectory, and the estimated error is much smaller than EKF. Therefore, compared with the conventional EKF, the proposed scheme can achieve a better performance when the target changes directions dynamically, which is more practical in actual applications.

To implement the KCF, the local sensor nodes need to exchange their message packets, which include three parts: node information vector $u_{i}$, information matrix $U_{i}$, and the average target state value $\bar{x}_{i}$. For 2-dimensional target tracking, the exchanged data amount almost is less than 50 bytes. Every active node will broadcast the information. In target tracking process, the average number of the active nodes is 


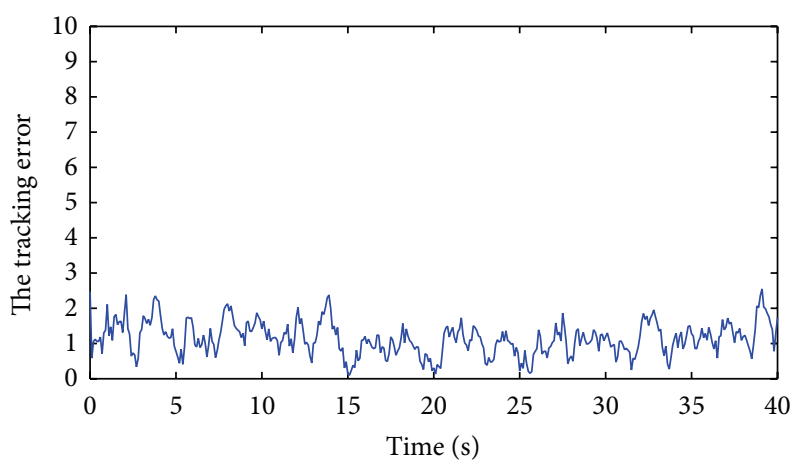

- KCF

FIGURE 3: Tracking accuracy by using the proposed method.

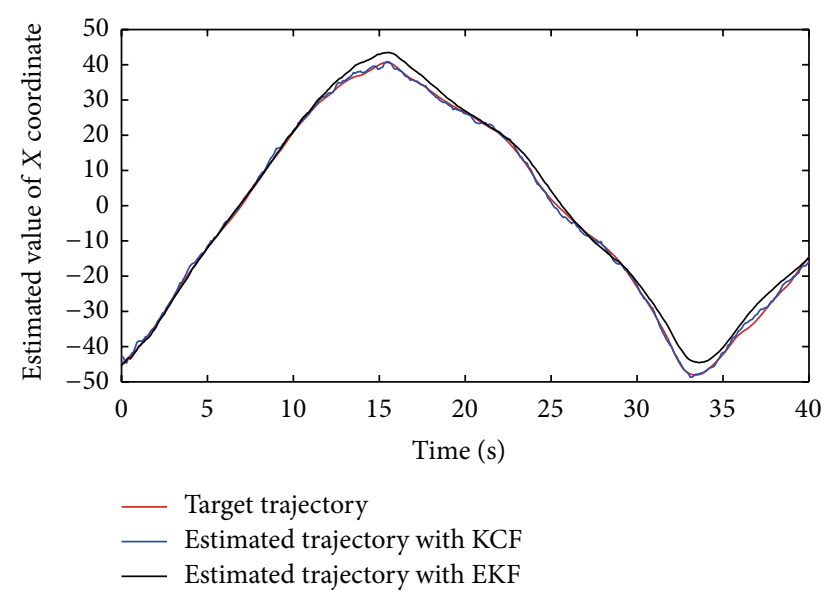

FIGURE 4: The comparison of the estimated value of $\mathrm{X}$ coordinate between KCF and EKF.

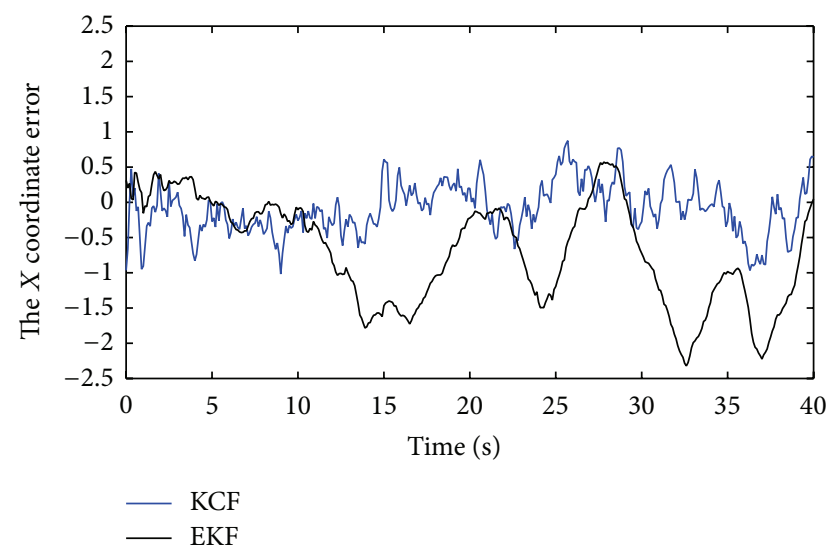

FIgURE 5: The comparison of the estimated error of $\mathrm{X}$ coordinate between KCF and EKF.

limited to 10 due to the application of node selection. Thus the increased complexity is tolerable in practical tracking scene.

To improve the energy efficiency, the sensor node selection algorithm is introduced into target tracking. As described in the introduction, the node selection algorithm can be classified into two categories: random selection and

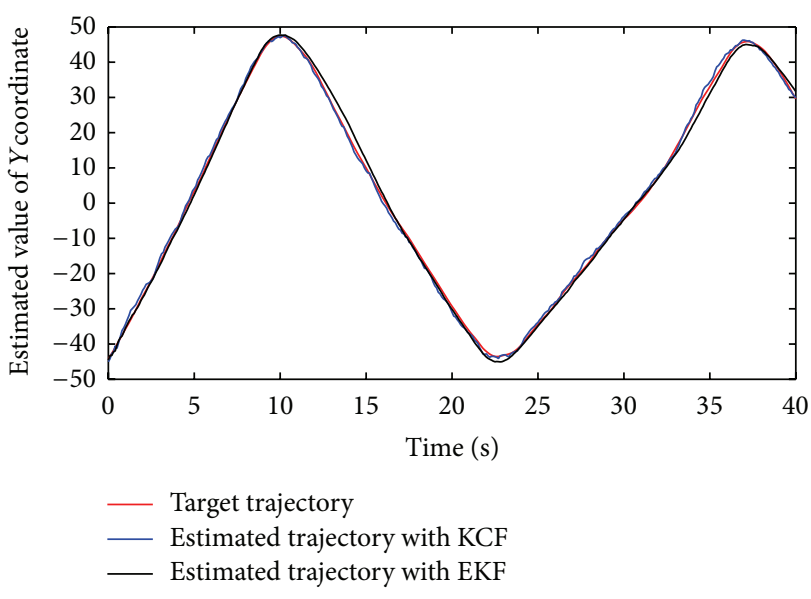

FIGURE 6: The comparison of the estimated value of $\mathrm{Y}$ coordinate between KCF and EKF.

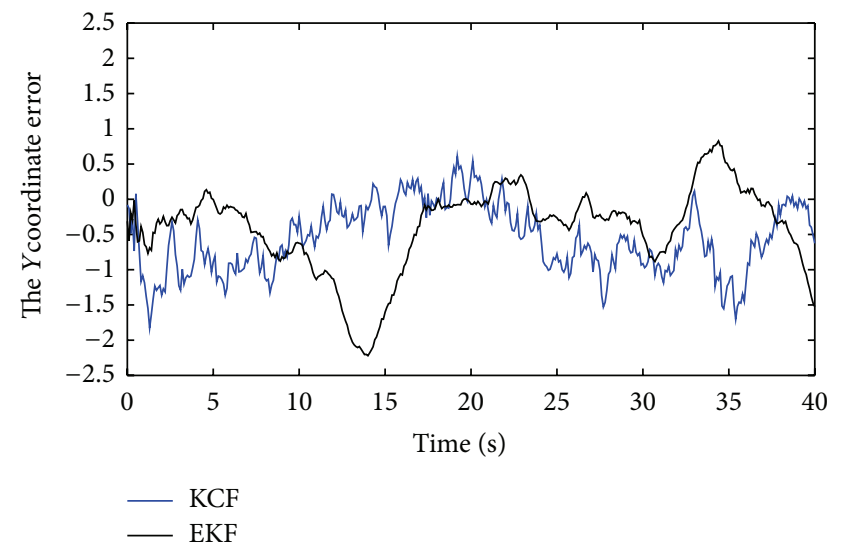

FIGURE 7: The comparison of the estimated error of $\mathrm{Y}$ coordinate between KCF and EKF.

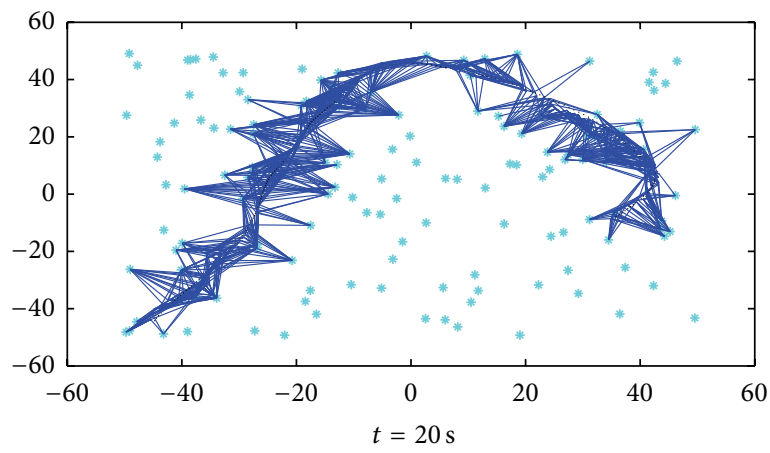

FIgURE 8: Target trajectory using random node selection with fixed number of sensor nodes for each sensing step.

adaptive selection. Figures 8 and 9 give the estimated target trajectories using random selection and adaptive selection, respectively. In these figures, the red dot denotes the estimated position and the blue line connects the sensor node to its estimation. From Figure 8, it is shown that the blue lines in Figure 8 are much denser than in Figure 9, which means 


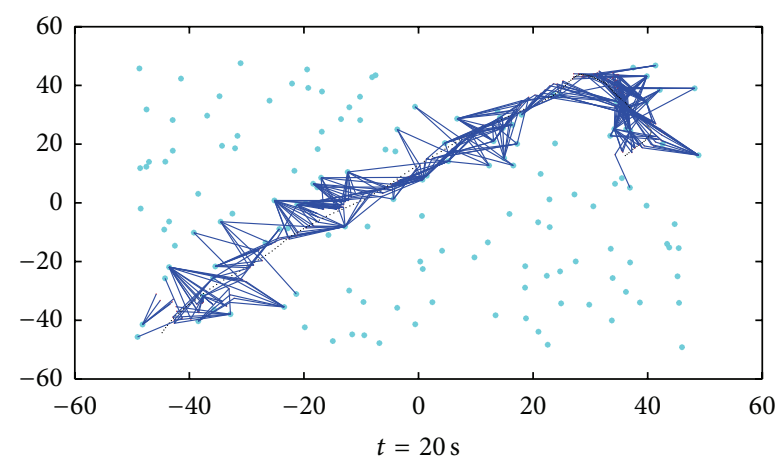

FIGURE 9: Target trajectory using the proposed adaptive node selection with various number of sensor nodes for each sensing step.

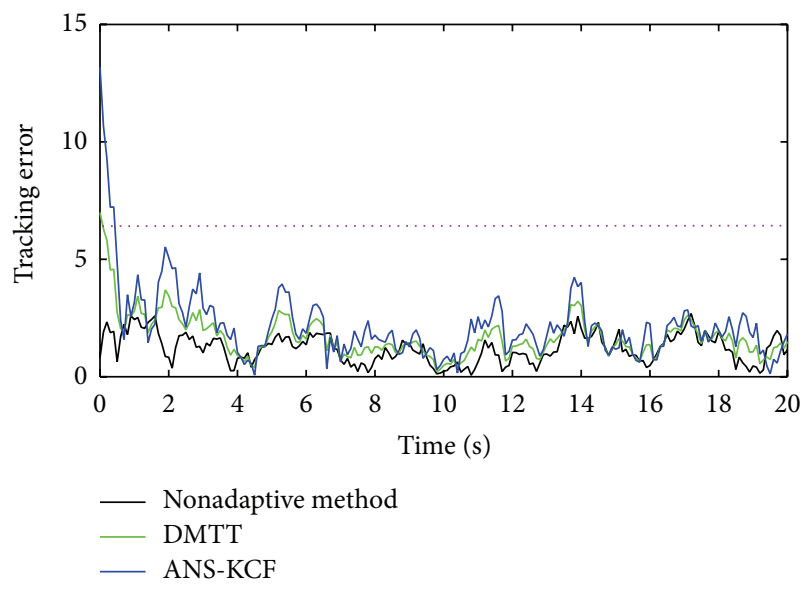

FIGURE 10: Tracking accuracy with nonadaptive, DMTT, and ANSKCF methods.

that the random selection algorithm uses more sensor nodes than the proposed adaptive node selection algorithm. But their tracking performance is similar. Thus some sensor nodes in random selection algorithm could not be necessary.

To represent this, Figure 10 displays the comparison of the tracking accuracy with nonadaptive and adaptive methods, DMTT algorithm, and the proposed ANS-KCF algorithm. As shown in Figure 10, nonadaptive algorithm indeed outperforms the nonadaptive methods in the tracking accuracy because the nonadaptive algorithm uses fixed sample frequency and has more sensor nodes that participate in target tracking. But the differences existing are minor. All the three methods can achieve the system requirement, but the proposed ANS-KCF algorithm can reduce the number of participating sensor nodes mostly, thus saving energy significantly.

Figures 11 and 12 show the number of tasking sensor nodes and the sampling interval of the three methods, respectively. For nonadaptive methods, the participating nodes' number and sampling interval are fixed. But for adaptive methods, in the beginning of tracking, just one sensor node found the target as shown in Figure 11. Thus just the smallest sampling interval was chosen to improve the tracking accuracy, as shown in Figure 12.

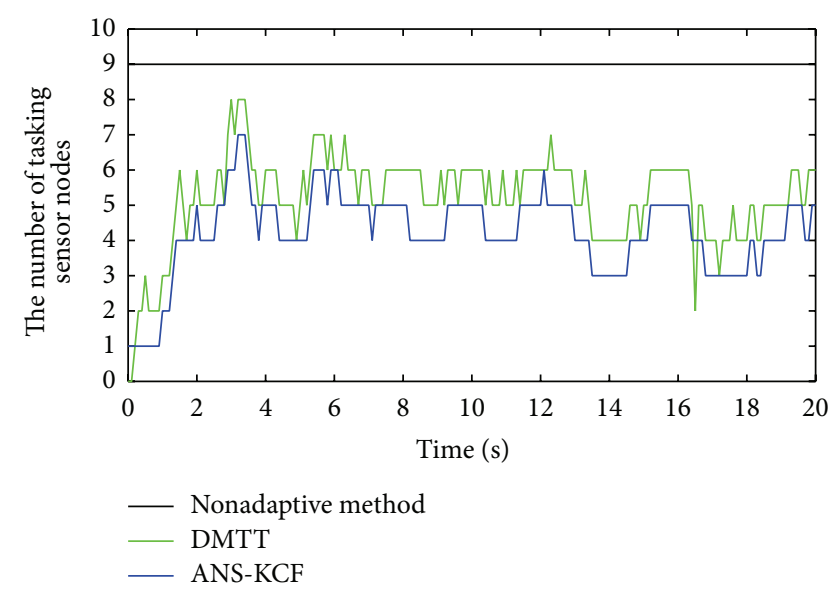

FIGURE 11: The number of tasking sensor nodes participating in the target tracking at different time for nonadaptive, DMTT, and ANSKCF methods.

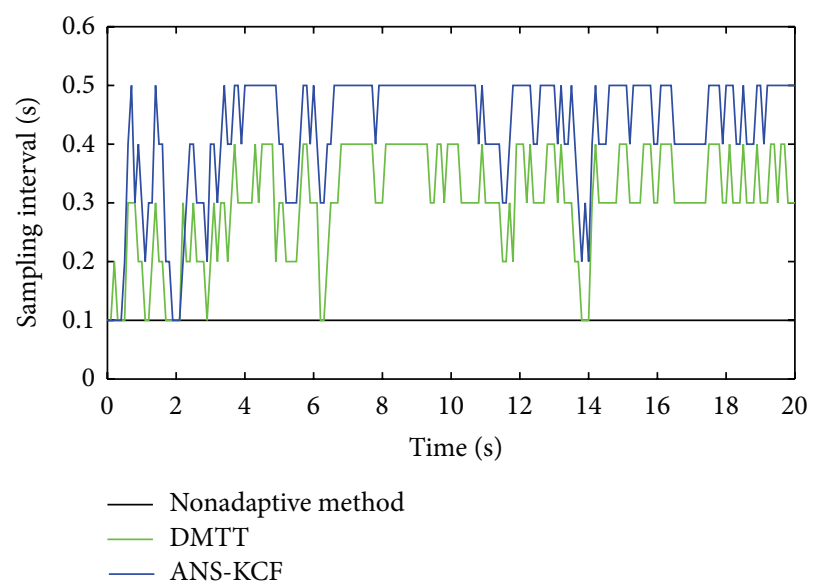

FIGURE 12: The sampling interval of tasking sensor nodes participating in the target tracking at different time for nonadaptive, DMTT, and ANS-KCF methods.

After that, the tracking accuracy is improved with the number of sensing nodes increasing. But the tracking process is tending towards stability with the tracking going on. Also from Figures 11 and 12, it is shown that the proposed algorithm ANS-KCF allows fewer nodes to participate in tracking and allows larger sample interval than DMTT, which illustrates that the proposed algorithm ANS-KCF has more energy consideration.

Finally the energy performance is evaluated. Figure 13 shows the energy consumption in target tracking using nonadaptive method with fixed number of active sensing nodes and uniform sampling interval, $\Delta t=0.1$, and the target tracking using DMTT and the proposed algorithm ANS-KCF. The comparison results show that the energy consumption is smallest when the proposed algorithm ANS-KCF was adopted because it uses fewer nodes and larger sample interval to realize tracking.

Compared to nonadaptive algorithm, the proposed adaptive algorithm has node selection procedure, which will 


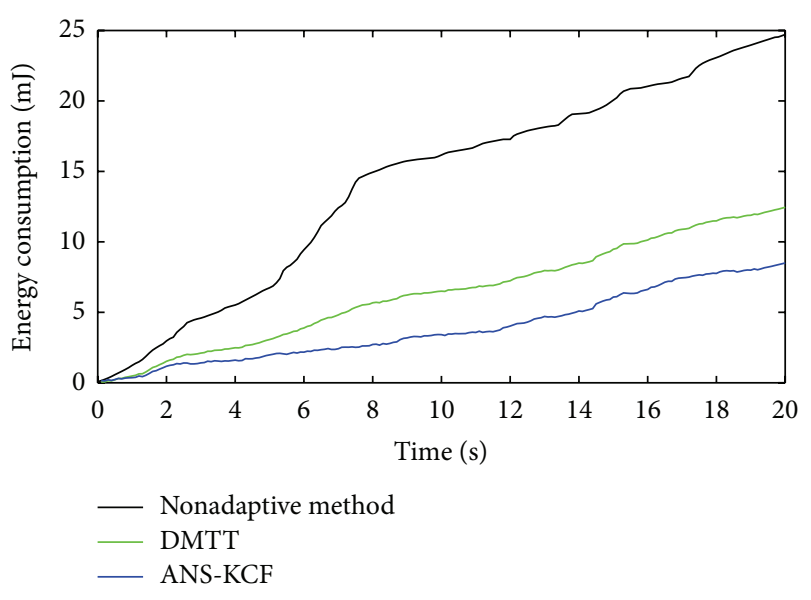

FIGURE 13: The comparison of the energy consumption for nonadaptive, DMTT, and ANS-KCF methods.

increase the implementation complexity. From the analysis of Section 3.2, the complexity of algorithm is $\left(N \cdot \log _{2}\left(\lambda_{\max }-\right.\right.$ $\left.\lambda_{\min }\right) / \varepsilon+N_{T}$ ), which mainly increases the computation consumption with polynomial order. Under the parameter setting of the simulation, the node selection can be implemented in real time. Compared to the saving energy, the computing consumption can be accepted.

\section{Conclusion and Future Works}

In this paper, we have proposed a novel adaptive node scheduling method for energy-efficient target tracking in wireless sensor networks. Firstly, the Kalman-consensus filter is improved to support the cooperative node tracking. Then the node scheduling problem with the energy and accuracy constraints is decomposed and analyzed by convex framework. The novelty of the proposed method lies in using index gradient rather than using brute research to decide the suitable sensor nodes. The method realizes the tradeoff between tracking accuracy and energy efficiency for resource-limited sensor networks. In our future work, we will focus on the scenario that the sensing range of a sensor will decay as energy consumption.

\section{Conflict of Interests}

The authors declare that there is no conflict of interests regarding the publication of this paper.

\section{Acknowledgments}

This work is partially supported by the National Natural Science Foundation of China (nos. 61003233, 61379111, and 61202342) and Specialized Research Fund for Doctoral Program of Higher Education (no. 20110162110042).

\section{References}

[1] J. Yick, B. Mukherjee, and D. Ghosal, "Wireless sensor network survey," Computer Networks, vol. 52, no. 12, pp. 2292-2330, 2008.

[2] P. Corke, T. Wark, R. Jurdak, W. Hu, P. Valencia, and D. Moore, "Environmental wireless sensor networks," Proceedings of the IEEE, vol. 98, no. 11, pp. 1903-1917, 2010.

[3] G. Simon, M. Maroti, A. Ledeczi et al., "Sensor network-based countersniper system," in Proceedings of the 2nd International Conference on Embedded Networked Sensor Systems (SenSys '04), pp. 1-12, Baltimore, Md, USA, November 2004.

[4] I. Vasilescu, K. Kotay, D. Rus et al., "Data collection, storage, and retrieval with an underwater sensor network," in Proceedings of the 3rd International Conference on Embedded Networked Sensor Systems, pp. 154-165, ACM, 2005.

[5] J. H. Huang, S. Amjad, and S. Mishra, "Cenwits: a sensor-based loosely coupled search and rescue system using witnesses," in Proceedings of the 3rd International Conference on Embedded Networked Sensor Systems, pp. 180-191, ACM, 2005.

[6] P. Zhang, C. M. Sadler, S. A. Lyon, and M. Martonosi, "Hardware design experiences in ZebraNet," in Proceedings of the 2nd International Conference on Embedded Networked Sensor Systems (SenSys '04), pp. 227-238, Baltimore, Md, USA, November 2004.

[7] O. Demigha, W.-K. Hidouci, and T. Ahmed, "On Energy efficiency in collaborative target tracking in wireless sensor network: a review," IEEE Communications Surveys and Tutorials, vol. 15, no. 3, pp. 1210-1222, 2013.

[8] K. J. Hintz, "A measure of the information gain attributable to cueing," IEEE Transactions on Systems, Man and Cybernetics, vol. 21, no. 2, pp. 434-442, 1991.

[9] F. Zhao, J. Shin, and J. Reich, "Information-driven dynamic sensor collaboration," IEEE Signal Processing Magazine, vol. 19, no. 2, pp. 61-72, 2002.

[10] J. Manyika and H. Durrant-Whyte, Data Fusion and Sensor Management: A Decentralized Information-Theoretic Approach, Prentice Hall PTR, Upper Saddle River, NJ, USA, 1995.

[11] R. R. Brooks, P. Ramanathan, and A. M. Sayeed, "Distributed target classification and tracking in sensor networks," Proceedings of the IEEE, vol. 91, no. 8, pp. 1163-1171, 2003.

[12] D. Li, K. D. Wong, Y. H. Hu, and A. M. Sayeed, "Detection, classification, and tracking of targets," IEEE Signal Processing Magazine, vol. 19, no. 2, pp. 17-29, 2002.

[13] H. Wang, G. Pottie, K. Yao, and D. Estrin, "Entropy-based sensor selection heuristic for target localization," in Proceedings of the 3rd International Symposium on Information Processing in Sensor Networks (IPSN '04), pp. 36-45, ACM, April 2004.

[14] J. Chen, K. Cao, K. Li, and Y. Sun, "Distributed sensor activation algorithm for target tracking with binary sensor networks," Cluster Computing, vol. 14, no. 1, pp. 55-64, 2011.

[15] H. Rowaihy, S. Eswaran, M. Johnson et al., "A survey of sensor selection schemes in wireless sensor networks," in Unattended Ground, Sea, and Air Sensor Technologies and Applications IX, vol. 6562 of Proceedings of SPIE, Orlando, Fla, USA, April 2007.

[16] D. Guo and X. Wang, "Dynamic sensor collaboration via sequential Monte Carlo," IEEE Journal on Selected Areas in Communications, vol. 22, no. 6, pp. 1037-1047, 2004.

[17] R. Tharmarasa, T. Kirubarajan, and M. L. Hernandez, "Largescale optimal sensor array management for multitarget tracking," IEEE Transactions on Systems, Man and Cybernetics Part C: Applications and Reviews, vol. 37, no. 5, pp. 803-814, 2007. 
[18] Y. E. M. Hamouda and C. Phillips, "Adaptive sampling for energy-efficient collaborative multi-target tracking in wireless sensor networks," IET Wireless Sensor Systems, vol. 1, no. 1, pp. $15-25,2011$.

[19] R. Olfati-Saber, "Distributed Kalman filtering for sensor networks," in Proceedings of the 46th IEEE Conference on Decision and Control, pp. 5492-5498, 2007.

[20] N. Ahmed, S. S. Kanhere, and S. Jha, "Probabilistic coverage in wireless sensor networks," in Proceedings of the IEEE 30th Anniversary Conference on Local Computer Networks, vol. 8, p. 681, IEEE, 2005.

[21] W. Xiao, S. Zhang, J. Lin, and C. K. Tham, "Energy-efficient adaptive sensor scheduling for target tracking in wireless sensor networks," Journal of Control Theory and Applications, vol. 8, no. 1, pp. 86-92, 2010.

[22] M. Bhardwaj and A. P. Chandrakasan, "Bounding the lifetime of sensor networks via optimal role assignments," in Proceedings of the IEEE information Conmmunication Conference (INFOCOM '02), pp. 1587-1596, New York, NY, USA, June 2002.

[23] T. Rappaport, Wireless Communication: Principles and Practice, Prentice Hall PTR, Upper Saddle River, NJ, USA, 2nd edition, 2001. 


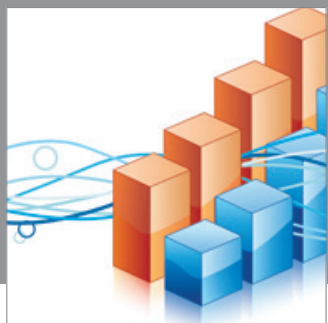

Advances in

Operations Research

mansans

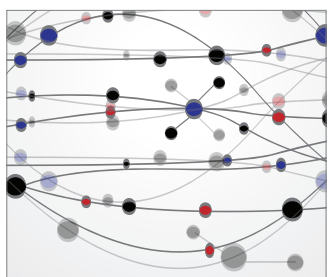

The Scientific World Journal
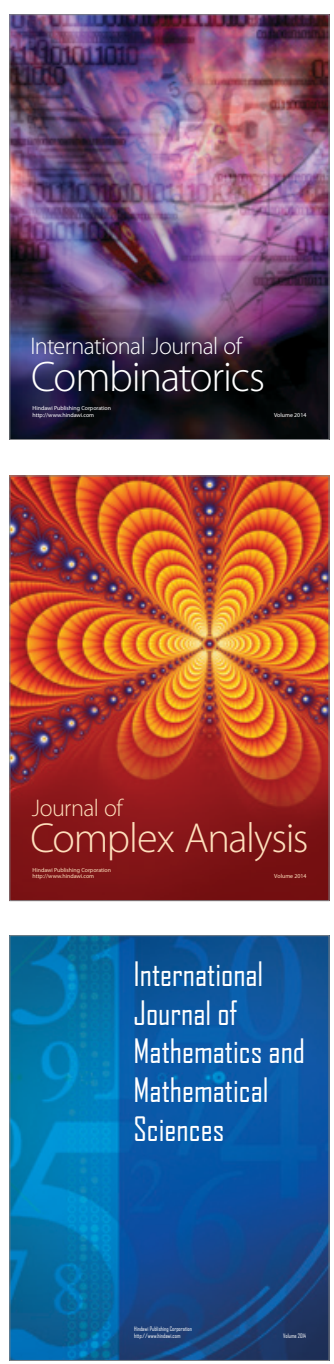
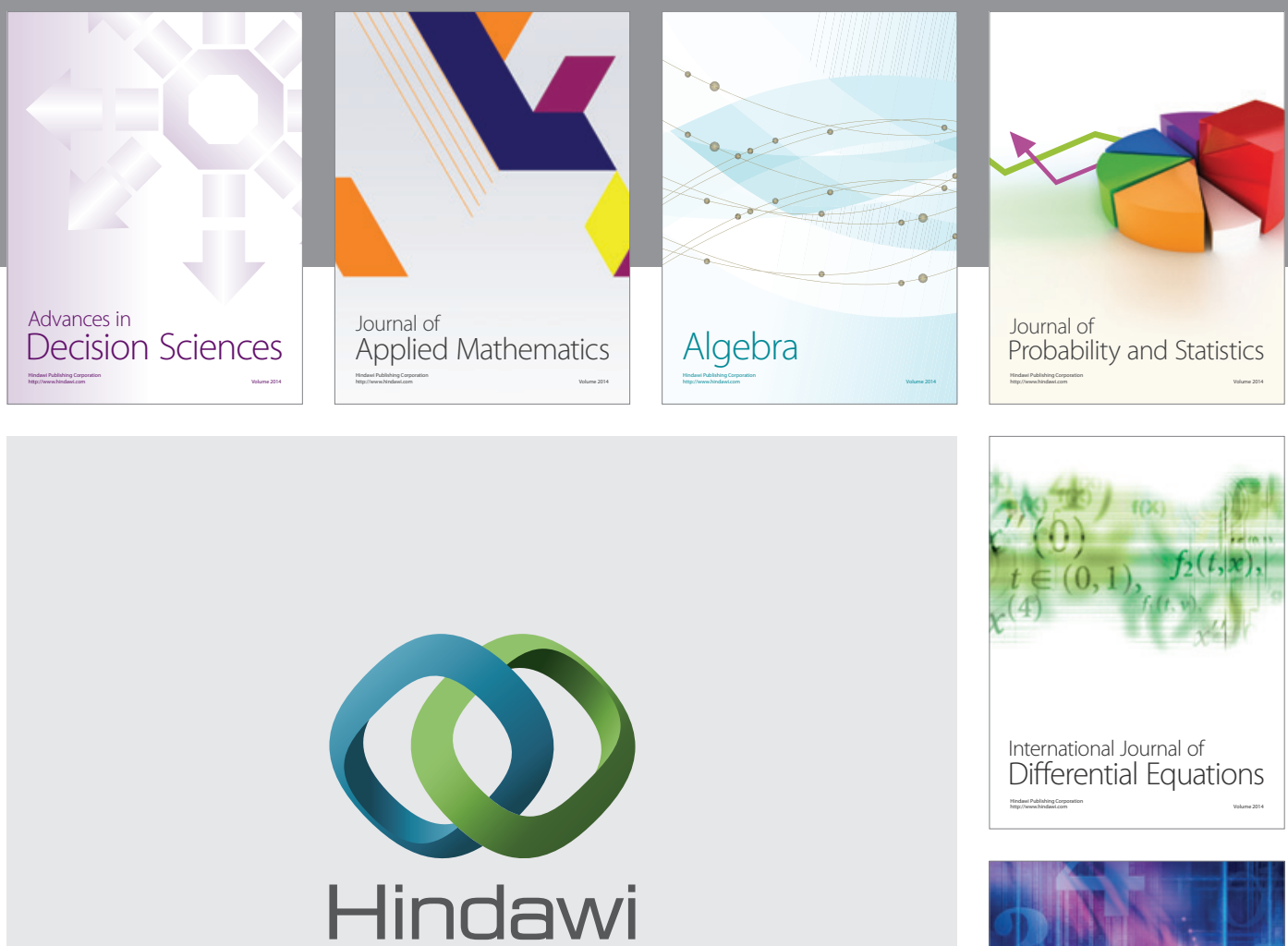

Submit your manuscripts at http://www.hindawi.com
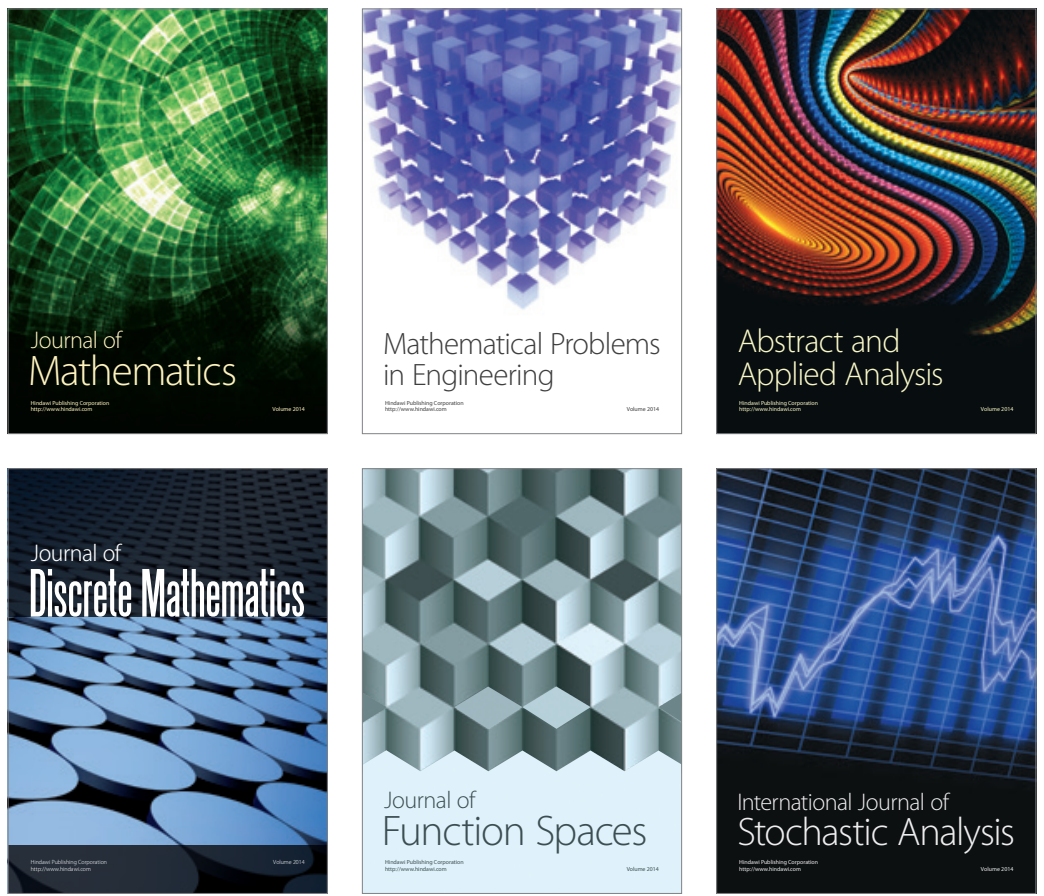

Journal of

Function Spaces

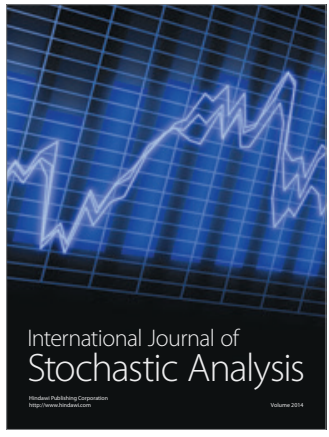

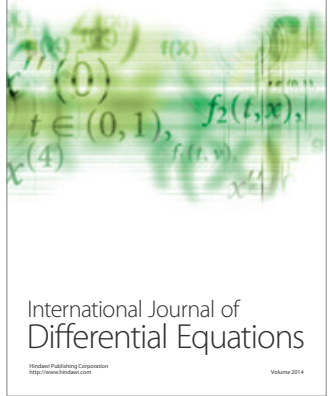
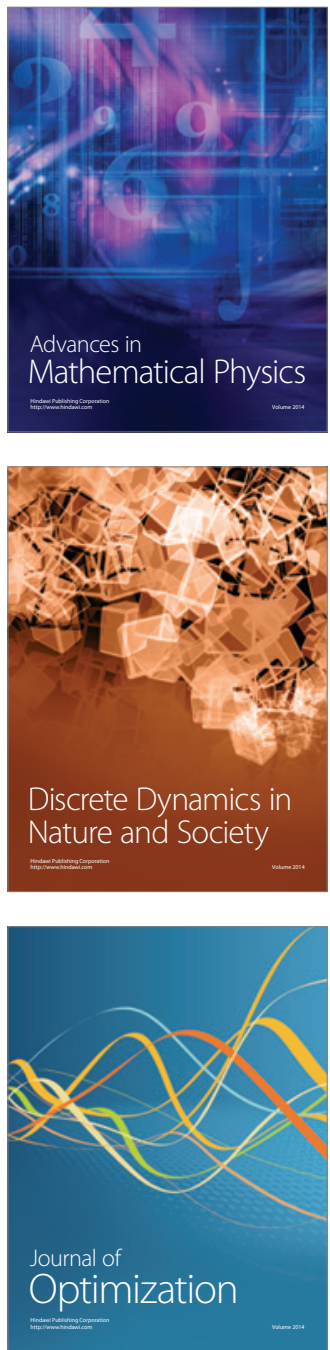